\title{
ON CONJUGACY OF MAXIMAL SUBALGEBRAS OF SOLVABLE LIE ALGEBRAS
}

\section{DAVID A. TOWERS}

\author{
Department of Mathematics and Statistics \\ Lancaster University \\ Lancaster LA1 4YF \\ England \\ d.towers@lancaster.ac.uk
}

\begin{abstract}
The purpose of this paper is to consider when two maximal subalgebras of a finite-dimensional solvable Lie algebra $L$ are conjugate, and to investigate their intersection.

Mathematics Subject Classification 2010: 17B05, 17B30, 17B40, 17B50.

Key Words and Phrases: Lie algebras, solvable, maximal subalgebra, conjugate, complement, chief factor.
\end{abstract}

Throughout $L$ will denote a finite-dimensional solvable Lie algebra over a field $F$. Let $x \in L$ and let ad $x$ be the corresponding inner derivation of $L$. If $F$ has characteristic zero suppose that $(\operatorname{ad} x)^{n}=0$ for some $n$; if $F$ has characteristic $p$ suppose that $x \in I$ where $I$ is a nilpotent ideal of $L$ of class less than $p$. Put

$$
\exp (\operatorname{ad} x)=\sum_{r=0}^{\infty} \frac{1}{r !}(\operatorname{ad} x)^{r} .
$$

Then $\exp (\operatorname{ad} x)$ is an automorphism of $L$. We shall call the group $\mathcal{I}(L)$ generated by all such automorphisms the group of inner automorphisms of 
$L$. More generally, if $B$ is a subalgebra of $L$ we denote by $\mathcal{I}(L: B)$ the group of automorphisms of $L$ generated by the $\exp (\operatorname{ad} x)$ with $x \in B$. Two subsets $U, V$ are conjugate under $\mathcal{I}(L: B)$ if $U=\alpha(V)$ for some $\alpha \in \mathcal{I}(L: B)$; they are conjugate in $L$ if they are conjugate under $\mathcal{I}(L)=\mathcal{I}(L: L)$.

If $U$ is a subalgebra of $L$, the centraliser of $U$ in $L$ is the set $C_{L}(U)=$ $\{x \in L:[x, u]=0\}$. In [1] Barnes showed that if $A$ is a minimal ideal of $L$ that is equal to its own centraliser in $L$, then $A$ is complemented in $L$ and all complements are conjugate under $\mathcal{I}(L: A)$. In [4] Stitzinger extended this result by finding necessary and sufficient conditions for two complements of an arbitrary minimal ideal of $L$ to be conjugate.

Theorem 0.1 ([4, Theorem 1]) Let A be a minimal ideal of the solvable Lie algebra $L$. Then there is a bijection between the set $\mathcal{M}$ of conjugacy classes of complements to $A$ under $\mathcal{I}(L: A)$ and the set $\mathcal{N}$ of complements to $A$ in $C_{L}(A)$ that are ideals of $L$.

Corollary 0.2 ([4, Corollary]) Suppose that $L$ is a solvable Lie algebra and let $M, K$ be complements to a minimal ideal $A$ of $L$. Then $M$ and $K$ are conjugate under $\mathcal{I}(L: A)$ if and only if $M \cap C_{L}(A)=K \cap C_{L}(A)$.

Clearly, such complements are maximal subalgebras of $L$. The purpose of this paper is to consider further when two maximal subalgebras of $L$ are conjugate, and to investigate their intersection.

Lemma 0.3 Let $L$ be a solvable Lie algebra, and let $M, K$ be two core-free maximal subalgebras of $L$. Then $M, K$ are conjugate under $\exp (a d a)=$ $1+$ ad a for some $a \in A$; in particular, they are conjugate in $L$.

Proof. Let $A$ be a minimal abelian ideal of $L$. Then $L=A \oplus M=A \oplus K$, $C_{L}(A)=A$ and $A$ is the unique minimal ideal of $L$, by [5, Lemma 1.3]. The result, therefore, follows from [3, Theorem 1.1].

If $U$ is a subalgebra of $L$, its core, $U_{L}$, is the largest ideal of $L$ contained in $U$.

Theorem 0.4 Suppose that $L$ is a solvable Lie algebra over a field $F$. If $F$ has characteristic $p$ suppose further that $L^{2}$ has nilpotency class less than $p$. Let $M, K$ be maximal subalgebras of $L$. Then $M$ is conjugate to $K$ in $L$ if and only if $M_{L}=K_{L}$. 
Proof. Suppose first that $M, K$ are conjugate in $L$, so that $K=\alpha(M)$ for some $\alpha \in \mathcal{I}(L)$. Then it is easy to see that $\exp (\operatorname{ad} x)\left(M_{L}\right)=M_{L}$ whenever $\exp (\operatorname{ad} x)$ is an automorphism of $L$, whence $K_{L}=\alpha\left(M_{L}\right)=M_{L}$.

Conversely, suppose that $M_{L}=K_{L}$. Then $M / M_{L}, K / M_{L}$ are corefree maximal subalgebras of $L / M_{L}$, and so are conjugate under $\mathcal{I}\left(L / M_{L}\right.$ : $\left.\left(L / M_{L}\right)^{2}\right)$, by Lemma 0.3. But now $M$ and $K$ are conjugate under $\mathcal{I}\left(L: L^{2}\right)$ by $[2$, Lemma 5$]$, and so are conjugate in $L$.

The above result does not hold for all solvable Lie algebras, as the following example shows.

EXAMPLE 0.1 Let $F$ be a field of characteristic $p$ and consider the Lie algebra $L=\left(\oplus_{i=0}^{p-1} F e_{i}\right) \dot{+} F x \dot{+} F y$ with $\left[e_{i}, x\right]=e_{i+1}$ for $i=0, \ldots, p-2$, $\left[e_{p-1}, x\right]=e_{0},\left[e_{i}, y\right]=i e_{i}$ for $i=0, \ldots, p-1,[x, y]=x$, and all other products zero. Let $C$ be a faithful completely reducible L-module. Since $L$ is monolithic with monolith $A=\oplus_{i=0}^{p-1} F e_{i}, C$ has a faithful irreducible submodule $B$. Let $X$ be the split extension of $B$ by $L$. Then $A+F x+F y$ and $A+F\left(x+e_{1}\right)+F y$ are maximal subalgebras of $X$, both of which have $A$ as their core. However, $B$ is the unique minimal ideal of $L$ and these subalgebras are not conjugate under inner automorphisms of the form $1+a d b$, $b \in B$. Since $B$ is the nilradical of $X$, defining other inner automorphisms is problematic.

Let $0=L_{0}<L_{1}<\ldots<L_{n}=L$ be a chief series for $L$ and let $M$ be a maximal subalgebra of $L$. Then there exists $k$ with $0 \leq k \leq n-1$ such that $L_{k} \subseteq M$ but $L_{k+1} \nsubseteq M$. Clearly $L=M+L_{k+1}$ and $M \cap L_{k+1}=L_{k}$; we say that the chief factor $L_{k+1} / L_{k}$ is complemented by $M$.

Theorem 0.5 Suppose that $L$ is a solvable Lie algebra over a field $F$. If $F$ has characteristic $p$ suppose further that $L^{2}$ has nilpotency class less than $p$. Let $A / B$ be a chief factor of $L$ that is complemented by a maximal subalgebra $M$ of $L$. If $K$ is conjugate to $M$ in $L$ then $K=\exp (a d a)(M)$ for some $a \in A$ and $M \cap K=\{m \in M:[m, a] \in M\}$.

Proof. We have that $L=A+M$ with $A^{2} \subseteq M_{L}, M^{2} \subseteq M_{L}, B \subseteq A \cap M_{L}$, and $M_{L}=K_{L}$. Clearly then $B \subseteq A \cap M_{L} \subseteq A$. Moreover, $A \neq A \cap M_{L}$ since $A \nsubseteq \nsubseteq M$. It follows that $B=A \cap M_{L}$ because $A / B$ is a chief factor. Thus

$$
\frac{A+M_{L}}{M_{L}} \cong \frac{A}{A \cap M_{L}}=\frac{A}{B},
$$


whence $\left(A+M_{L}\right) / M_{L}$ is a minimal abelian ideal of $L / M_{L}$. Lemma 0.3 implies that $K / M_{L}=\exp \left(\operatorname{ad}\left(a+M_{L}\right)\right)\left(M / M_{L}\right)$ for some $a \in A$.

Now $[L, A] \subseteq B$ or $[L, A]=A$. The former implies that $[L, A] \subseteq M_{L}$, contradicting the fact that $\left(A+M_{L}\right) / M_{L}$ is self-centralising in $L / M_{L}$, by [5, Lemma 1.4]. Hence $A=[L, A] \subseteq L^{2}$, and so $\exp (\operatorname{ad} a)$ is defined. If $x \in$ $\exp (\operatorname{ad} a)(M)$ then $x+M_{L}=\exp (\operatorname{ad} a)(m)=m+[m, a]+M_{L} \in K / M_{L}$ for some $m \in M$, whence $x \in K$. Since $\exp (\operatorname{ad} a)$ is an automorphism of $L$ we must have $K=\exp (\operatorname{ad} a)(M)$.

Finally we have $(M \cap K) / M_{L}=\left(M / M_{L}\right) \cap\left(K / M_{L}\right)=C_{\left(M / M_{L}\right)}\left(a+M_{L}\right)$ by [5, Lemma 1.5]. We infer that $m \in M \cap K \Leftrightarrow[m, a] \in M_{L} \Leftrightarrow[m, a] \in M$.

Theorem 0.5 gave a characterisation of the intersection of two conjugate maximal subalgebras of $L$. Finally we consider the intersection of two nonconjugate maximal subalgebras of $L$.

Theorem 0.6 Suppose that $L$ is a solvable Lie algebra over a field $F$. Let $M, K$ be maximal subalgebras of $L$, and suppose that $K_{L} \nsubseteq M_{L}$. Then $M \cap K$ is a maximal subalgebra of $M$.

Proof. We have that $K_{L} \nsubseteq \nsubseteq M$, so $L=M+K_{L}=M+K$. If $K=K_{L}$ then $L / K \cong M /(M \cap K)$ and the result is clear. So suppose that $K \neq K_{L}$. Let $A / K_{L}$ be a minimal ideal of $L / K_{L}$. Then $L / K_{L}=A / K_{L} \oplus K / K_{L}$, from [5, Lemma 1.4], giving $A \cap K=K_{L}$. Also, $A=A \cap\left(M+K_{L}\right)=A \cap M+K_{L}$, whence

$$
\frac{A}{K_{L}}=\frac{A \cap M+K_{L}}{K_{L}} \cong \frac{A \cap M}{K_{L} \cap M},
$$

showing that $(A \cap M) /\left(K_{L} \cap M\right)$ is a minimal ideal of $M /\left(K_{L} \cap M\right)\left(\cong L / K_{L}\right)$ and $A \cap M$ is a minmal ideal of $M$. Now

$$
\begin{aligned}
& \operatorname{dim}\left(\frac{M}{M \cap K}\right) \geq \operatorname{dim}\left(\frac{A \cap M+M \cap K}{M \cap K}\right)=\operatorname{dim}\left(\frac{A \cap M}{K_{L} \cap M}\right) \\
= & \operatorname{dim}\left(\frac{A}{K_{L}}\right)=\operatorname{dim}\left(\frac{L}{K}\right)=\operatorname{dim}\left(\frac{M+K}{K}\right)=\operatorname{dim}\left(\frac{M}{M \cap K}\right) .
\end{aligned}
$$

It follows that $M=A \cap M+M \cap K$ which yields the result.

Corollary 0.7 Suppose that $L$ is a solvable Lie algebra over a field F. If $F$ has characteristic $p$ suppose further that $L^{2}$ has nilpotency class less than $p$. Let $M, K$ be maximal subalgebras of $L$ that are not conjugate in $L$. Then $M \cap K$ is a maximal subalgebra of at least one of $M, K$. 
Corollary 0.8 Suppose that $L$ is a solvable Lie algebra and let $M, K$ be complements to a minimal ideal $A$ of $L$ that are not conjugate in $L$. Then $M \cap K$ is a maximal subalgebra of both $M$ and $K$.

Proof. By Theorem 0.1, both $M_{L}$ and $K_{L}$ are complements to $A$ in $C_{L}(A)$. Since $M_{L} \neq K_{L}$ we have $M_{L} \nsubseteq K_{L}$ and $K_{L} \nsubseteq M_{L}$. The result now follows from Theorem 0.6.

\section{References}

[1] D.W. Barnes, 'On the cohomology of soluble Lie algebras', Math. Z. 101 (1967), 343-349.

[2] D.W. Barnes, 'On Cartan subalgebras of Lie algebras', Math. Z. 101 (1967), 350-355.

[3] D.W. Barnes and M.L. Newell, 'Some Theorems on saturated homnomorphs of soluble Lie algebras', Math. Z. 115 (1970), 179-187.

[4] E.L. Stitzinger, 'On a theorem of D.W. Barnes', Canad. Math. Bull. 14 (1971), 583-584.

[5] D.A. Towers, 'On Lie algebras in which modular pairs of subalgebras are permutable', J. Algebra 68 (1981), 369-377. 